\title{
Jamming Transition in Granular Systems
}

\author{
T. S. Majmudar, ${ }^{1}$ M. Sperl, ${ }^{1}$ S. Luding, ${ }^{2}$ and R. P. Behringer ${ }^{1}$ \\ ${ }^{1}$ Department of Physics, Duke University, Box 90305, Durham, North Carolina 27708, USA \\ ${ }^{2}$ Technische Universiteit Delft, DelftChemTech, Particle Technology, Nanostructured Materials, \\ Julianlaan 136, 2628 BL Delft, The Netherlands
}

(Received 23 October 2006; published 29 January 2007)

\begin{abstract}
Recent simulations have predicted that near jamming for collections of spherical particles, there will be a discontinuous increase in the mean contact number $Z$ at a critical volume fraction $\phi_{c}$. Above $\phi_{c}, Z$ and the pressure $P$ are predicted to increase as power laws in $\phi-\phi_{c}$. In experiments using photoelastic disks we corroborate a rapid increase in $Z$ at $\phi_{c}$ and power-law behavior above $\phi_{c}$ for $Z$ and $P$. Specifically we find a power-law increase as a function of $\phi-\phi_{c}$ for $Z-Z_{c}$ with an exponent $\beta$ around 0.5 , and for $P$ with an exponent $\psi$ around 1.1. These exponents are in good agreement with simulations. We also find reasonable agreement with a recent mean-field theory for frictionless particles.
\end{abstract}

DOI: 10.1103/PhysRevLett.98.058001

PACS numbers: 45.70. $-\mathrm{n}, 64.60 .-\mathrm{i}, 83.80 . \mathrm{Fg}$

A solid, in contrast to a fluid, is characterized by mechanical stability that implies a finite resistance to shear and isotropic deformation. While such stability can originate from long-range crystalline order, there is no general agreement on how mechanical stability arises for disordered systems, such as molecular and colloidal glasses, gels, foams, and granular packings [1]. For a granular system, in particular, a key question concerns how stability occurs when the packing fraction $\phi$ increases from below to above a critical value $\phi_{c}$ for which there are just enough contacts per particle $Z$ to satisfy the conditions of mechanical stability. In recent simulations on frictionless systems it was found that $Z$ exhibits a discontinuity at $\phi_{c}$ followed by a power-law increase for $\phi>\phi_{c}$ [2-5]. The pressure is also predicted to increase as a power law above $\phi_{c}$.

A number of recent theoretical studies address jamming, and we note work that may be relevant to granular systems. Silbert, O'Hern et al. have shown in computer simulations of frictionless particles [2-4] that (a) for increasing $\phi, Z$ increases discontinuously at the transition point from zero to a finite number, $Z_{c}$, corresponding to the isostatic value (needed for mechanical stability), (b) for both two- and three-dimensional systems, $Z$ is expected to continue increasing as $\left(\phi-\phi_{c}\right)^{\beta}$ above $\phi_{c}$, where $\beta=0.5$, (c) the pressure $P$ is expected to grow above $\phi_{c}$ as $\left(\phi-\phi_{c}\right)^{\psi}$, where $\psi=\alpha_{f}-1$ in the simulations, and $\alpha_{f}$ is the exponent for the interparticle potential. More recent simulations by Donev et al. for hard spheres in three dimensions found a slightly higher value for $\beta, \beta \approx 0.6$, in maximally random jammed packings [5]. It is interesting to note that a model for foam exhibits quite similar behavior for $Z$ [6]. Henkes and Chakraborty [7] constructed a mean-field theory of the jamming transition in 2D based on entropy arguments. These authors predict power-law scaling for $P$ and $Z$ in terms of a variable $\alpha$, which is the pressure derivative of the entropy. By eliminating $\alpha$, one obtains an algebraic relation between $P$ and $Z-Z_{c}$ from these predictions, which we present below in the context of our data.
While the simulations agree among themselves at least qualitatively, so far, these novel features have not been identified in experiments. Hence, it is crucial to test these predictions experimentally. In the following, we present experimental data for $Z$ and $P$ vs $\phi$, based on a method that yields accurate determination of the contacts and identifies power laws in $Z$ and $P$ for a two-dimensional experimental system of photoelastic disks. By measuring both $P$ and $Z$, we can also obtain a sharper value for the critical packing fraction $\phi_{c}$, for the onset of jamming, and we can test the model of Henkes and Chakraborty.

The relevant simulations have been carried out predominantly for frictionless particles. For real frictional particles there will clearly be some differences. For instance, in the isostatic limit, $Z$ equals 4 for frictionless disks, whereas for frictional disks, $Z$ is around 3, depending on the system details [8]. Other predictions such as specific critical exponents may also need modification. However, one might hope that the observed experimental behavior, in particular, critical exponents, might be similar to that for frictionless particles if the frictional forces are typically small relative to the normal forces. Indeed, in recent experiments, the typical intergrain frictional forces in a physical granular system were found to be only about $10 \%$ of the normal forces [9].

Figure 1(a) shows a schematic of the apparatus. We use a bidisperse mixture (80\% small and $20 \%$ large particles) of approximately 3000 polymer (PSM-4) photoelastic (birefringent under stress) disks with diameter 0.74 or $0.86 \mathrm{~cm}$. This ratio preserves a disordered system. The disks have Young's modulus of $4 \mathrm{MPa}$, and a static coefficient of friction of 0.85 . The model granular system is confined in a biaxial test cell $(42 \mathrm{~cm} \times 42 \mathrm{~cm}$ with two movable walls) which rests on a smooth Plexiglas sheet. The displacements of the walls can be set very precisely with stepper motors. The linear displacement step size used in this experiment is $40 \mu \mathrm{m}$, which is approximately $0.005 \mathrm{D}$, where $D$ is the average diameter of the disks. The defor- 
(a)

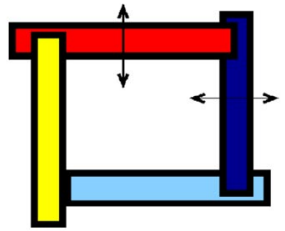

(d)

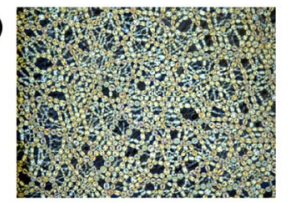

(b)

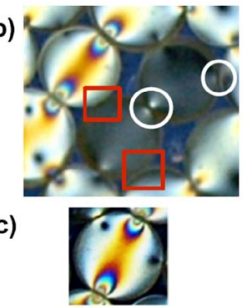

(e)

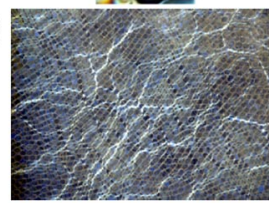

FIG. 1 (color online). (a) Schematic cross section of biaxial cell experiment (not to scale). Two walls can be moved independently to obtain a desired sample deformation. (b) Examples of contacts and particles that are either close but not actually in contact, or contacts with very small forces. Circles show true contacts, squares show false apparent contacts. (c) Image of a single disk at the typical resolution of the experiment. (d) Sample image of highly jammed/compressed state and (e) almost unjammed state.

mation $\delta$ per particle is less than $1 \%$ in the compressed state. The setup is horizontal and placed between crossed circular polarizers. It is imaged from above with an $8 \mathrm{MP}$ CCD color camera which captures roughly 1200 disks in the center of the cell, enabling us to visualize the stress field within each disk (Fig. 1). We then obtain good measurements of the vector contact forces (normal and tangential $=$ frictional components) [9].

We also use the particle photoelasticity to accurately determine the presence or absence of contacts between particles. In numerical studies one can use a simple overlap criterion to determine contacts: a contact occurs if the distance between particle centers is smaller than the sum of the particle radii. However, in experimental systems, a criterion based solely on the particle centers is susceptible to relatively large errors which include false positives [Fig. 1(b) (squares)] as well as false negatives (circles). As seen in Fig. 1(b), the contacts through which there is force transmission appear as source points for the stress pattern. Further details are given in Sec. I of the supplementary material [10].

We use two protocols to produce different packing fractions: we either compress the system from an initially stress-free state, or decompress the system until the end state is essentially a stress-free state. The results for both protocols are the same within error bars above $\phi_{c}$; below jamming, the data for $Z$ obtained by compression are a few percent below those for decompression. Below, we will present decompression data. Figures 1(d) and 1(e) show the initial highly stressed state and the end state after decompression, respectively. After each decompression step, we apply tapping to relax stress in the system. This could be seen as roughly analogous to the annealing process invoked in some simulations. Two images are captured at

each state: one without polarizers to determine the disk centers and one with polarizers to record the stress.

The average $Z$ can be computed either by counting only the force bearing disks or by counting all the disks including rattlers which do not contribute to the mechanical stability of the system. We consider as rattlers, all the disks which have less than two contacts. For the number of rattlers beyond the transition point we find an exponential decrease with $\phi-\phi_{c}$; hence, a divergence in the number of rattlers at $\phi_{c}$ is not indicated by the data.

We next compute the Cauchy stress tensor for each disk, $\sigma_{i j}=\frac{1}{2 A} \sum\left(F_{i} x_{j}+F_{j} x_{i}\right) ; P$ is the trace of this tensor. Here, $A$ is the Voronoi area for the given disk, and the sum is taken over contacts for a given disk. We then compute the average of the pressure over the ensemble of disks in the system. For the data presented below, we performed two sets of experiments: one with a larger range, $0.8390 \leq \phi \leq 0.8650$, and also larger step size, $\Delta \phi=$ 0.016 , and, after the jamming region was identified, a second set at a finer scale with $0.840745 \leq \phi \leq$ 0.853312 , with a step size, $\Delta \phi=0.000324$.

The inset in Fig. 2 shows data for $Z$ over a broad range of $\phi$ [with rattlers (stars); without (squares)]. These data show a significant rise in $Z$ at the jamming transition.

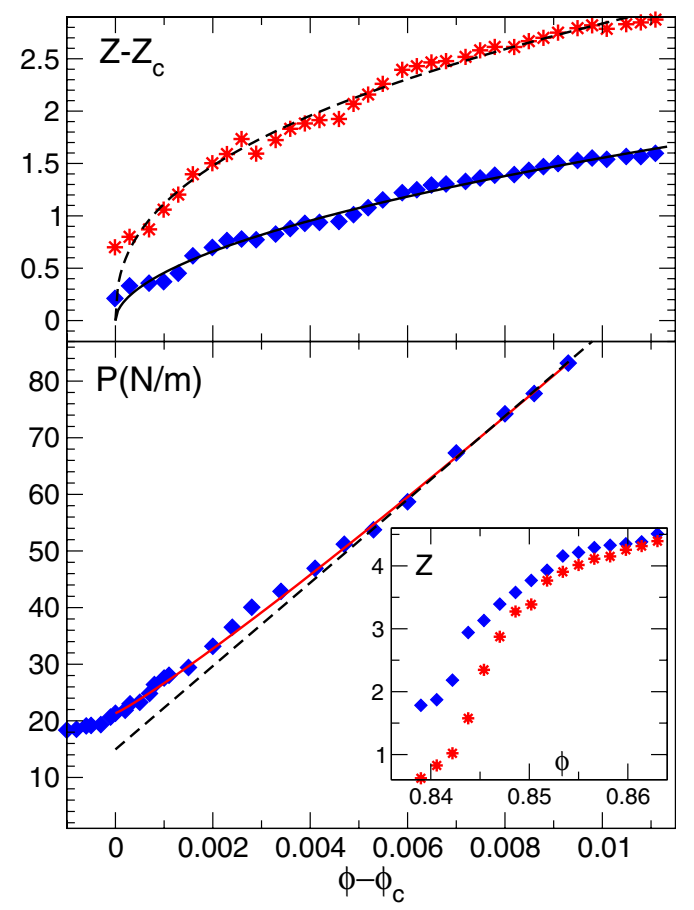

FIG. 2 (color online). Average contact number and pressure at the jamming transition. Top and bottom panels show $Z-Z_{c}$ and $P$ vs $\phi-\phi_{c}$, respectively, with rattlers included (stars) or excluded (diamonds). Dashed and full curves in the top panel give power-law fits $\left(\phi-\phi_{c}\right)^{\beta}$ with $\beta=0.495$ and 0.561 for the case with and without rattlers, respectively. Full curve in the lower panel gives the fit $\left(\phi-\phi_{c}\right)^{\psi}$ with $\psi=1.1$; dashed line shows a linear law for comparison. Inset: $Z$ vs $\phi$ for a larger range in $\phi$. 
While this rise is not sharply discontinuous, it occurs over a very small range in $\phi$. At higher $\phi$, the variations of the curves are similar with and without rattlers. At lower $\phi$, their behavior differs: The values of $Z$ drop lower for the case with rattlers. The pressure $P\left(\phi-\phi_{c}\right)$ in Fig. 2 shows a flat background below jamming, and then a sharp positive change in slope at a well defined $\phi$. The pressure is not identically zero below jamming for similar reasons that the jump in $Z$ is not perfectly sharp, as discussed below.

To compare these experimental results to predictions above $\phi_{c}$, we carry out least squares fits of $Z-Z_{c}$ and $P$ to $\phi-\phi_{c}$. These fits depend on the choice of $\phi_{c}$, which has some ambiguity due to the rounding; the data allow a range from around 0.840 to 0.843 . In fact, $\phi_{c}$ can be determined in several ways: the point where $Z$ reaches 3 , the point where $P$ begins to rise above the background, etc. (cf. Ref. [10]). We show results of these fits in Fig. 2, starting with the upper panel, which shows power-law fits $\left(Z-Z_{c}\right) \propto\left(\phi-\phi_{c}\right)^{\beta}$. The fitted exponent $\beta$ depends on the choice of $\phi_{c}$ but the variation is small without rattlers, $0.494 \leq \beta \leq 0.564$, and somewhat larger with rattlers, $0.363 \leq \beta \leq 0.525$. The details for several different specific fits are given in Sec. II of Ref. [10]. The point $\phi_{c}=$ 0.84220 where $P$ rises above the background level is used in Fig. 2, and yields a consistent fit for both $P$ and $Z$. The point where $Z$ reaches 3 for the case without rattlers agrees with the previous case to within $\delta \phi_{c}=0.0005$, and the exponents are quite similar. Comparing with the simulations for frictionless particles, we find that our values of $\beta \approx 0.55$ for the data without rattlers are larger than the value of 0.5 reported in [2,3], but smaller than those of Donev et al. [5] obtaining 0.6 in 3D. In contrast, for a model of frictional disks under shear, Aharonov and Sparks [11] obtain the much lower value of 0.36 . However, a direct comparison is not possible to the present case of jamming under isotropic conditions.

Figure 2 shows the variation of $P$ with $\phi$ in the lower panel, indicating a clear transition at $\phi_{c}=0.8422 \pm$ 0.0005 . For this choice of $\phi_{c}, P$ increases as $P \propto(\phi-$ $\left.\phi_{c}\right)^{\psi}$ with $\psi=1.1 \pm 0.05$ above $\phi_{c}$. This value of $\psi$ pertains to a fit over the full range $\phi \geq \phi_{c}$ of Fig. 2; a larger exponent would be obtained if the fit range were limited to very close to $\phi_{c}$. This value is close to the value $\psi=1.0$ found $[2,3]$ for a linear force law, and this linear law is indicated as a dashed line in Fig. 2. One expects such a linear force law (with a logarithmic correction) for ideal disks, but direct mechanical calibration of the force law for the cylinders is closer to $\delta^{3 / 2}$ (see Ref. [10]). This rather high exponent for the force law is attributable to the small asperities, which influence the force law for small deformations. However, the photoelastic response is detectable only for $\delta>150 \mu \mathrm{m}$, and for such $\delta$ 's, the force law is close to locally linear in $\delta$.

From the $P$ vs $\phi$ data, we can also obtain the bulk modulus, $B=-A \partial P / \partial A$, where $A$ is the area enclosed by the system boundaries. Since, $\phi=A_{p} / A$, where $A_{p}$ is the (presumably fixed) area occupied by the disks $B=$ $\phi \partial P / \partial \phi$. Then, $B \propto\left(\phi-\phi_{c}\right)^{\psi-1}$, which gives a weak pressure variation of $B$ above $\phi_{c}$. We note that anomalous results for the bulk modulus have been observed in acoustical experiments by Jia, and discussed by Makse et al. [12], where the bulk modulus near $\phi_{c}$ varied faster with $P$ than was previously expected because of changes in $Z$.

Since $P$ in Fig. 2 corresponds closely to expectations for a linear force law, we performed a computer simulation for a polydisperse system of 1950 particles with a linear force law $\left(k_{n}=10^{5} \mathrm{~N} / \mathrm{m}\right)$ without friction; details can be found in [13]. In Fig. 3 the results are shown for a larger range in density than done in earlier studies. All the data in Fig. 3 can be fitted with a single value for the transition density of $\phi_{c}=0.84005$. While the average overlap per particle (equivalent of the deformation $\delta$ for physical particles) is clearly linear in $\phi$, the pressure $P$ is not: $P$ increases faster than linear with an exponent close to the one found in the experiment. $Z$ is also consistent with a power-law exponent close to 0.5 . With the rattlers included, $Z$ at $\phi_{c}, Z_{c}=3.94$, is slightly below the isostatic value of 4 for a frictionless system of disks.

To connect with the predictions of Henkes and Chakraborty [7], we consider $P-P_{c}$ vs $Z$. The prediction from their Eq. (10) is equivalent to $\left(P-P_{c}\right) / P_{c}=u-$ $\left[\left(4 u^{2}+1\right)^{1 / 2}-1\right] / 2$, where $u=C\left(Z-Z_{c}\right)$ and $C=\epsilon / \alpha_{c}$

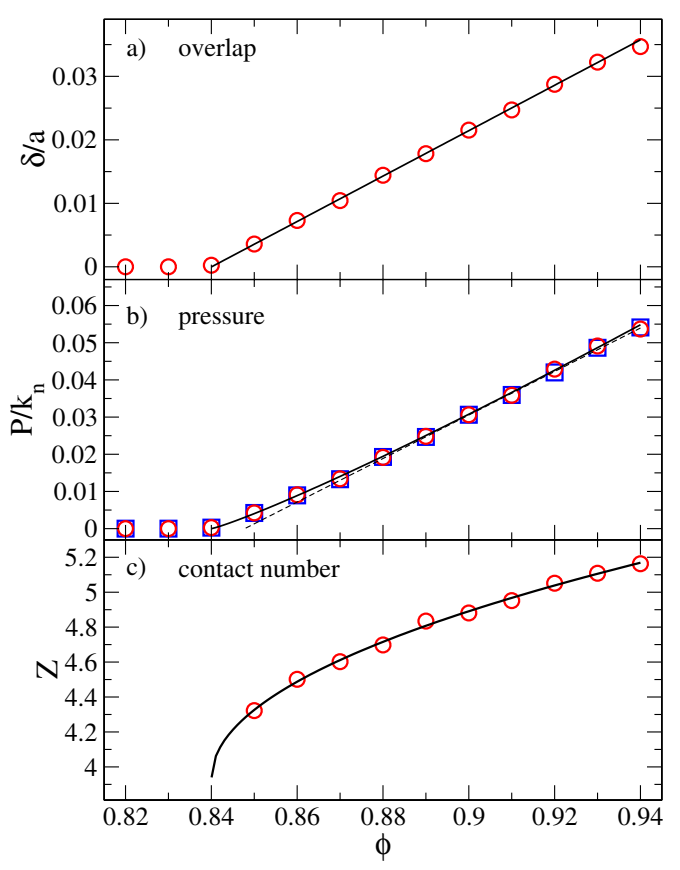

FIG. 3 (color online). Results from new computer simulations. For all fits $\phi_{c}=0.84005$. (a) Average overlap per particle in units of the mean particle radius is linear in $\phi-\phi_{c}$. (b) $P$ obtained from the Cauchy stress tensor (circles) and the force on the walls (squares) satisfy a power law $\left(\phi-\phi_{c}\right)^{\Psi}$ with $\psi=$ 1.13; dashed line shows a linear law for comparison. (c) $Z$ (rattlers included) exhibits a power law $Z-Z_{c} \propto\left(\phi-\phi_{c}\right)^{\beta}$ with $Z_{c}=3.94$ and $\beta=0.5015$. 


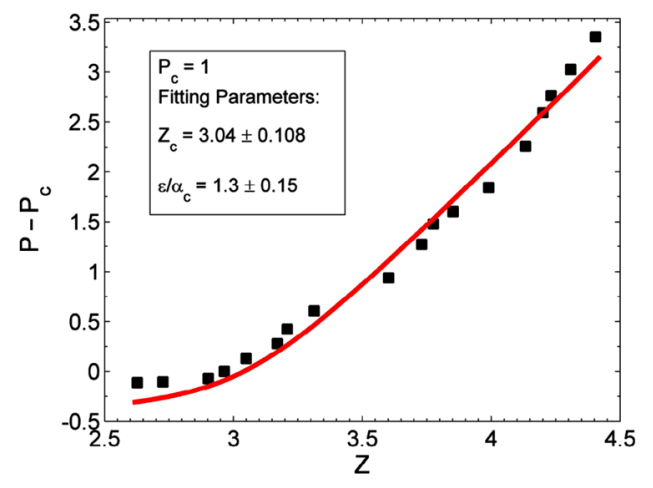

FIG. 4 (color online). Pressure vs $Z$; experimental data and a fit to the model of Henkes and Chakraborty [7]. In this fit, the constant $C$ defined in the text is treated as an adjustable parameter. The other fitting parameter is $Z_{c}$.

is a system-dependent constant. Thus, $\epsilon$ is a measure of the grain elasticity, and $\epsilon=0$ corresponds to completely rigid grains. Also, $\alpha_{c}$ is the critical value for $\alpha$. In fitting to this form, we may adjust $P_{c}$, (within reason) $Z_{c}$, and $C$. In Fig. 4 we find reasonable although not perfect agreement with this prediction (above $\phi_{c}$ ), and obtain $Z_{c}=3.04$, which is close to the isostatic value $Z_{c}=3$.

We now turn to the rounding that we observe in $Z$ quite close to the transition, and the background pressure that we obtain near $\phi_{c}$. One possible explanation is the friction between the disks and the Plexiglas base. This could help freeze in contact forces and contacts. However, a simple estimate of the upper bound for the friction with the base shows that this cannot be a significant effect, at least as regards the pressure background. To obtain an estimated upper bound for the base friction on $P$, we assume that base friction can support intergrain contact forces corresponding to the maximum base frictional force per grain, $F_{f}=$ $\mu_{\mathrm{ba}} m g=2.8 \times 10^{-3} \mathrm{~N}$, where $m$ is the mass of a grain and $\mu_{\mathrm{ba}}<1$ is the friction between a particle and the base. Assuming $Z$ interparticle contacts and one particle-base contact per grain, we estimate the resulting upper bound on the perturbation to the pressure as $\delta P \simeq\left(Z F_{f} R\right) /\left(\pi R^{2}\right) \simeq$ $0.22 Z \mathrm{~N} / \mathrm{m}$, where $R$ is a disk radius. Since $Z \simeq 3$, this pressure is almost 2 orders of magnitude too small to be of relevance. An additional issue concerns the anisotropy that is induced during compression or expansion by the apparatus. This induced anisotropy is difficult to avoid and/or relax close to $\phi_{c}$ even in the simulation, but it remains small. It is visible in Fig. 1(e), where a weak array of force chains tends to slant from lower left to upper right. Among other reasons, the anisotropy can be induced by wall friction due to the confining lateral boundaries of the biaxial apparatus.

We conclude by noting that these experiments, the first of which we are aware, demonstrate the critical nature of jamming in a real granular material. Our results take advantage of the high accuracy in contact number $Z$ that is afforded when the particles are photoelastic. $Z$ shows a very rapid rise at a packing density $\phi_{c}=0.8422$. The fine resolution in density allows us to see that the transition is not as sharply discontinuous under the present experimental conditions as in the computer simulation. Above $\phi_{c}, Z$ and $P$ follow power laws in $\phi-\phi_{c}$ with respective exponents $\beta$ of 0.5 to 0.6 and $\psi \approx 1$. 1 . The values for both $\beta$ and $\psi$ are consistent with recent simulation results for frictionless particles. In addition, we find reasonable agreement with a mean-field model of the granular jamming transition, again for frictionless particles. These results suggest that effects of friction on jamming are likely modest, although perhaps not ignorable. That jamming in the experiment occurs over a narrow, but finite range in $\phi$ seems mostly to be caused by small residual shear stresses that are induced by interactions with the walls confining the sample (not the base supporting the particles). The ability of a small amount of shear to affect the jamming transition is interesting, and points to the need for a deeper understanding of the effects of anisotropy.

This work was supported by No. NSF-DMR0137119, NSF-DMR0555431, NSF-DMS0244492, the U.S.-Israel Binational Science Foundation No. 2004391, and No. DFG-SP714/3-1. We thank E. Aharonov, B. Chakraborty, D. J. Durian, M. van Hecke, C. S. O'Hern, and S. Torquato for helpful discussions.

[1] A. J. Liu and S.R. Nagel, Jamming and Rheology: Constrained Dynamics on Microscopic and Macroscopic Scales (Taylor \& Francis, New York, 2001).

[2] L. E. Silbert, D. Ertaş, G. S. Grest, T. C. Halsey, and D. Levine, Phys. Rev. E 65, 031304 (2002).

[3] C. O'Hern, S. A. Langer, A. J. Liu, and S. R. Nagel, Phys. Rev. Lett. 88, 075507 (2002).

[4] C. O'Hern, L. E. Silbert, A. J. Liu, and S. R. Nagel, Phys. Rev. E 68, 011306 (2003).

[5] A. Donev, S. Torquato, and F. H. Stillinger, Phys. Rev. E 71, 011105 (2005).

[6] D. J. Durian, Phys. Rev. Lett. 75, 4780 (1995).

[7] S. Henkes and B. Chakraborty, Phys. Rev. Lett. 95, 198002 (2005).

[8] S. Alexander, Phys. Rep. 296, 65 (1998).

[9] T. S. Majmudar and R. P. Behringer, Nature (London) 435, 1079 (2005).

[10] See EPAPS Document No. E-PRLTAO-98-020705 for additional information for methods and data fits. For more information on EPAPS, see http://www.aip.org/ pubservs/epaps.html.

[11] E. Aharonov and D. Sparks, Phys. Rev. E 60, 6890 (1999).

[12] H. A. Makse, N. Gland, D. L. Johnson, and L. M. Schwartz, Phys. Rev. Lett. 83, 5070 (1999); X. Jia, C. Caroli, and B. Velicky, Phys. Rev. Lett. 82, 1863 (1999).

[13] M. Madadi, O. Tsoungui, M. Lätzel, and S. Luding, Int. J. Solids Struct. 41, 2563 (2004). 